\title{
Extension of Healthy Life Expectancy Through Measures Against Visual Impairment
}

\author{
YOSHIMUNE HIRATSUKA* \\ *Department of Ophthalmology, Juntendo University Graduate School of Medicine, Tokyo, Japan
}

\begin{abstract}
Japan's health system has received the world's highest rating from the WHO in 2000, but that is the story so far. Japan is facing serious problems, which is the sharp increase in the elderly population. The population ratio of the elderly continues to increase until 2042, and aging population ratio will exceed $40 \%$ in 2065 . Then it is the growth of medical expenditure that will cause enormous problems in the future. Extension of healthy life expectancy (HLE) is the key, and shortening the difference between lifespan and HLE is a present task in Japan. In order to extend HLE, it is necessary to shorten the period of reduction of degree of independence and bedridden, that is, requiring support and long-term care. And, the point is essential care prevention. There is a strong association between visual impairment and reasons for requiring long-term care / support. Measures against visual impairment will help to extend HLE.
\end{abstract}

Key words: healthy life expectancy (HLE), visual impairment, aging society, long-term care, vision related quality of life

\section{Japan's health system was No.1 in the world}

Japan's health system has received the world's highest rating from the WHO in 2000, and its degree of attainment and performance are the top level in the world ${ }^{1)}$. However, that is the story so far and Japan is facing serious problems in the future, which is the sharp increase in the elderly population. Japan has exceeded super aged society level already in 2010, now has entered the ultra aging society ahead of the world. Moreover, the trend of this aging will continue in the future. The population ratio of the elderly continues to increase until 2042 , and aging population ratio will exceed $40 \%$ in 2065. Then it is the growth of medical expenditure that will cause enormous problems in the future, since compare to younger patients, medical expenses of elderly people are tremendously high.

\section{Japan's national health promotion measures}

How is Japan trying to face this problem? Japan's national health promotion measures have been deployed to several degrees since 1978. In 1978, each citizen began with having awareness of protecting your own health by yourself, and since 1988, emphasis was placed on the diffusion of exercise habits. From 2000 on, it has proceeded with the trend of prevention of lifestyle-related disease development and severity prevention, which is well-known as metabolic syndrome. And now, in the decade from 2013, extension of healthy life expectancy (HLE) is the key. HLE is the concept that WHO proposed in 2000, and it refers to the period that we can live with full health or perfect health. So shortening the difference between normal lifespan and HLE is a present task in Japan.

Yoshimune Hiratsuka

Department of Ophthalmology, Juntendo University Graduate School of Medicine

2-1-1 Hongo, Bunkyo-ku, Tokyo 113-8421, Japan

TEL: +81-3-3813-3111 E-mail: yoshi-h@tkf.att.ne.jp

〔Received Dec. 20, 2018〕〔Accepted Jan. 11, 2019〕

Copyright (C) 2019 The Juntendo Medical Society. This is an open access article distributed under the terms of Creative Commons Attribution License (CC BY), which permits unrestricted use, distribution, and reproduction in any medium, provided the original source is properly credited. doi: 10.14789/jmj. 2019.65.JMJ18-LN04 


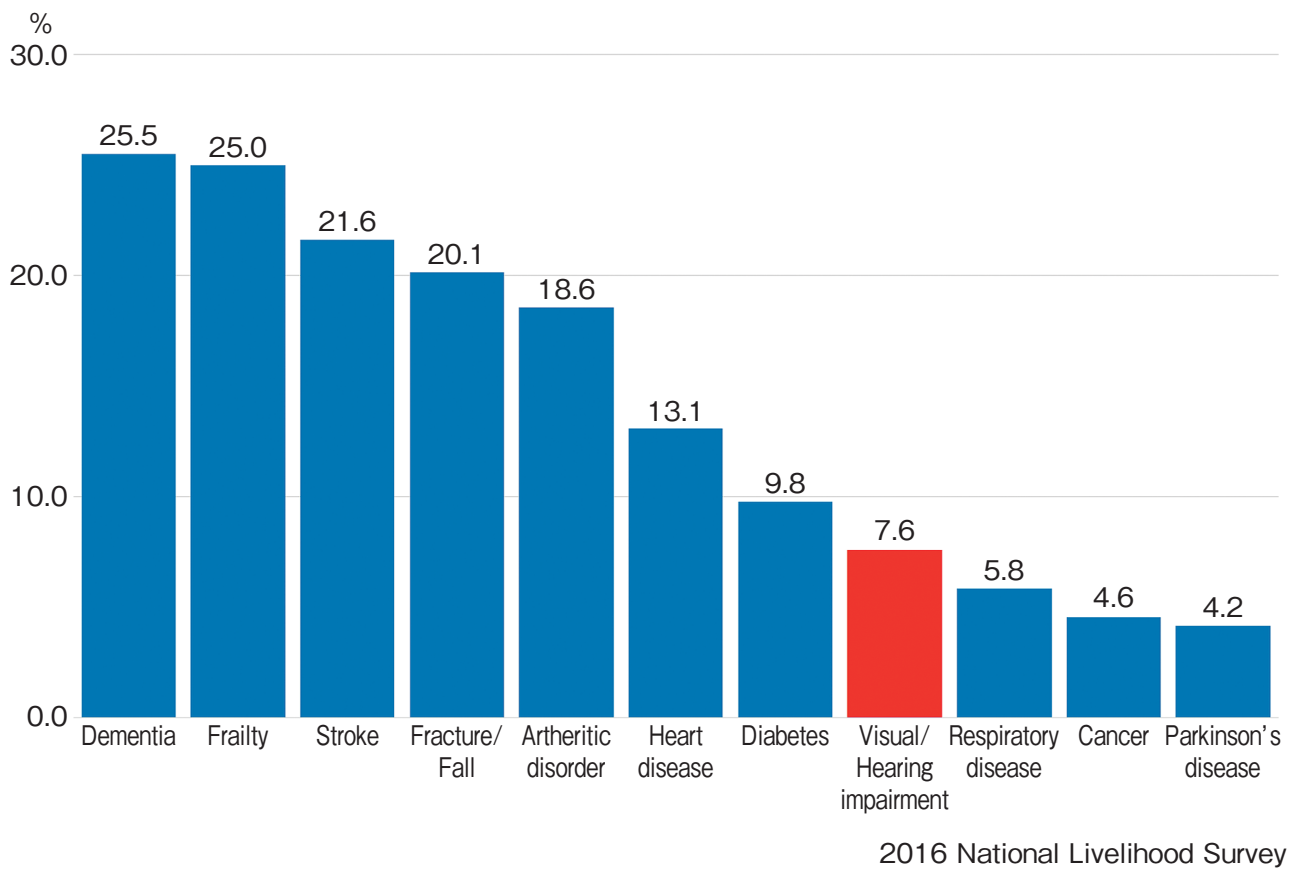

Figure-1 Causes of the requiring long-term care / support (2016)

\section{Extension healthy life expectancy through measures against visual impairment}

In order to extend HLE, it is necessary to shorten the period of reduction of degree of independence and bedridden, that is, requiring support and requiring long-term care. And, the point is essential care prevention. So, what are the causes of the need for long-term care? The causes in 2016 are as follows; dementia (26\%), frailty (25\%), stroke (22\%), falls/fractures (20\%), arthritic disorders (19\%), heart diseases (13\%), diabetes (10\%), visual and hearing impairment (8\%) and others (Figure1 ). Most of these causes are closely related to visual impairment. For example, it is clarified that people with visual impairment are lower in cognitive level than those without vision ${ }^{2}$. Vision-related QOL (VRQOL) and worse eye visual acuity are significantly associated with physical inactivity ${ }^{3)}$. Fundus examination can contribute to prevention of stroke
/ heart disease development. Higher VRQOL will reduce the frequency of falls ${ }^{4)}$. There is a strong association between visual impairment and reasons for requiring long-term care / support. Therefore, measures against visual impairment will help to extend healthy life expectancy, and in this regard, it is necessary to enlighten society.

\section{Reference}

1) World Health Organization, 2000: The World health report: 2000: Health systems: improving performance.

2) Maharani A, Dawes P, Nazroo J, Tampubolon G, Pendleton N; Sense-Cog WP1 group: Visual and hearing impairments are associated with cognitive decline in older people. Age Ageing, 2018; 47: 575-581.

3) Inoue S, Kawashima M, Hiratsuka Y, et al: Assessment of physical inactivity and locomotor dysfunction in adults with visual impairment. Sci Rep, 2018; 8: 12032.

4) Niihata K, Fukuma S, Hiratsuka Y, et al: Association between vision-specific quality of life and falls in community-dwelling elderly adults: LOHAS. PLoS One, 2018; 13: e0195806. 